Santa Clara University

Scholar Commons

Electrical Engineering

School of Engineering

$12-29-2008$

\title{
Secondary electron imaging of embedded defects in carbon nanofiber via interconnects
}

\author{
Makoto Suzuki \\ Yusuke Ominami \\ Takashi Sekiguchi \\ Cary Y. Yang \\ Santa Clara University, cyang@scu.edu
}

Follow this and additional works at: https://scholarcommons.scu.edu/elec

\section{Recommended Citation}

M. Suzuki, Y. Ominami, T. Sekiguchi, and C.Y. Yang, "Secondary electron imaging of embedded defects in carbon nanofiber via interconnects," Applied Physics Letters 93, 263110 (3 pp) (2008). https://doi.org/10.1063/1.3063053

Copyright (C) 2013 American Institute of Physics Publishing. Reprinted with permission.

This Article is brought to you for free and open access by the School of Engineering at Scholar Commons. It has been accepted for inclusion in Electrical Engineering by an authorized administrator of Scholar Commons. For more information, please contact rscroggin@scu.edu. 


\title{
Secondary electron imaging of embedded defects in carbon nanofiber via interconnects
}

\author{
Makoto Suzuki, ${ }^{1,2, a), b)}$ Yusuke Ominami, ${ }^{3, b)}$ Takashi Sekiguchi, ${ }^{1,2}$ and Cary Y. Yang ${ }^{3}$ \\ ${ }_{1}^{1}$ Graduate School of Pure and Applied Sciences, University of Tsukuba, Tsukuba, Ibaraki 305-8571, Japan \\ ${ }^{2}$ National Institute for Materials Science, Tsukuba, Ibaraki 305-0044, Japan \\ ${ }^{3}$ Center for Nanostructures, Santa Clara University, Santa Clara, California 95053, USA
}

(Received 30 November 2008; accepted 11 December 2008; published online 31 December 2008; publisher error corrected 7 January 2009)

\begin{abstract}
Carbon nanofiber $(\mathrm{CNF})$ via interconnect test structures are fabricated with the bottom-up process proposed by Li et al. [Appl. Phys. Lett. 82, 2491 (2003)] for next-generation integrated circuit technology. Critical defects in the interconnect structure are examined using scanning electron microscopy. It is shown that secondary electron signal with optimized incident beam energy is useful for detecting embedded defects, including unexposed CNF plugs and voids in the dielectric layer. The defect imaging mechanisms are elucidated based on beam-induced charging of the specimen surface. (C) 2008 American Institute of Physics. [DOI: 10.1063/1.3063053]
\end{abstract}

There are two major concerns in interconnect technology for the ever-shrinking feature sizes in integrated circuits, namely, electromigration ${ }^{1}$ in metallic interconnect materials such as aluminum and copper under large current density and the increase in electrical resistivity due to grain boundary and surface scatterings. ${ }^{2}$ One attempt to overcome such difficulties is the introduction of stable carbon nanostructures including carbon nanotubes (CNTs) (Ref. 3) and carbon nanofibers $(\mathrm{CNFs}){ }^{4} \mathrm{Li}$ et al. ${ }^{4}$ proposed a bottom-up fabrication process of vertical CNF interconnect using plasmaenhanced chemical vapor deposition (PECVD), making it possible to alleviate the difficulty of high-aspect-ratio contact hole fabrication expected in conventional lithography-based (i.e., top-down) approach. In this letter, we present a scanning electron microscopy (SEM) inspection technique for undesirable defects in the CNF interconnect structure fabricated using the bottom-up approach. Detection mechanisms of unexposed CNFs and embedded voids in intervia dielectrics are discussed based on the phenomenological theory of beam-induced specimen charging.

The bottom-up fabrication process employed in this study is schematically shown in Fig. 1(a). A 30-nm-thick titanium ( $\mathrm{Ti})$ is deposited on a silicon $(\mathrm{Si})$ substrate as the base contact layer. Subsequently a 35 -nm-thick nickel $(\mathrm{Ni})$ catalyst layer is deposited. During the PECVD growth, the heated Ni layer is turned into particles, which determine the position and diameter of the CNFs. The particle size can be controlled by the Ni layer thickness and microstructure. ${ }^{5}$ The as-grown CNFs are vertically aligned and freestanding on the substrate [Fig. 1(b)]. Silicon dioxide $\left(\mathrm{SiO}_{2}\right)$ embedding the CNFs to insulate and strengthen the vertical via arrays is then deposited using tetraethylorthosilicate (TEOS) CVD, followed by chemical-mechanical polishing (CMP) to expose the CNF plugs and to form electrical contact with the upper metal layer. The resulting thickness of the $\mathrm{SiO}_{2}$ layer is $5 \mu \mathrm{m}$. SEM imaging is performed in a field-emission scan-

\footnotetext{
a) Author to whom correspondence should be addressed. Electronic mail: suzuki-makoto@naka.hiatchi-hitec.com.

b) Permanent address: Hitachi High-Technologies Corp., Hitachinaka, Ibaraki 312-8504, Japan.
}

ning electron microscope (Hitachi S-4800), equipped with an in-column secondary electron (SE) detector for efficient lowenergy (i.e., less than several tens of eV) electron detection.

A typical cross section of a CNF via interconnect is shown in Fig. 1(c), which is prepared using $40 \mathrm{keV} \mathrm{Ga}^{+}$ion beam milling. As can be seen, incomplete $\mathrm{SiO}_{2}$ filling is found, resulting in voids in the layer. This is possibly due to the lack of TEOS gas in the area surrounded by the $\mathrm{SiO}_{2}$ grains and/or nonvertical CNFs. Locating the voids by cross sectioning the sample is a time-consuming process; thus it is worthwhile to have a detecting method using nondestructive SEM. Another defect type is short CNFs, which are not exposed even after CMP, leading to an electrical failure of the corresponding via plug. While the direct resistivity measurement performed using current-sensing probes would be useful, ${ }^{6}$ faster and more efficient SEM inspection is preferable in order to screen out the unexposed CNFs.

Figures 2(a)-2(j) show a series of SE images of a polished $\mathrm{SiO}_{2}$ top surface with changing the beam energy $(E)$ from $30 \mathrm{keV}$ to $100 \mathrm{eV}$. The energy range can be subdivided into three regions: region I $(E<1.0 \mathrm{keV})$, where the exposed CNFs are imaged as bright spots, region II $(1.5 \mathrm{keV}$

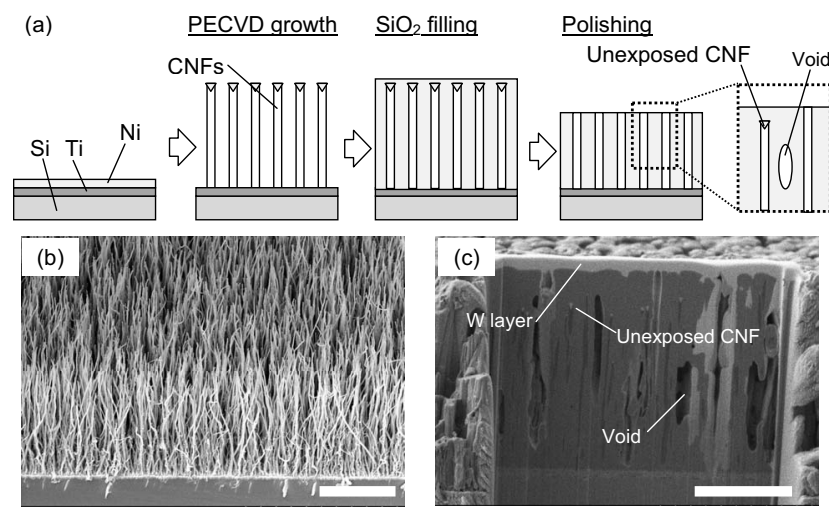

FIG. 1. (a) Schematics of the bottom-up fabrication process for vertical CNF interconnects. (b) SEM image of the as-grown CNF forest. (c) SEM image of the cross section of vertical CNF interconnects embedded in $\mathrm{SiO}_{2}$ prepared by focused $\mathrm{Ga}^{+}$ion beam milling. Scale bars in (b) and (c) are 10 and $3 \mu \mathrm{m}$, respectively. 

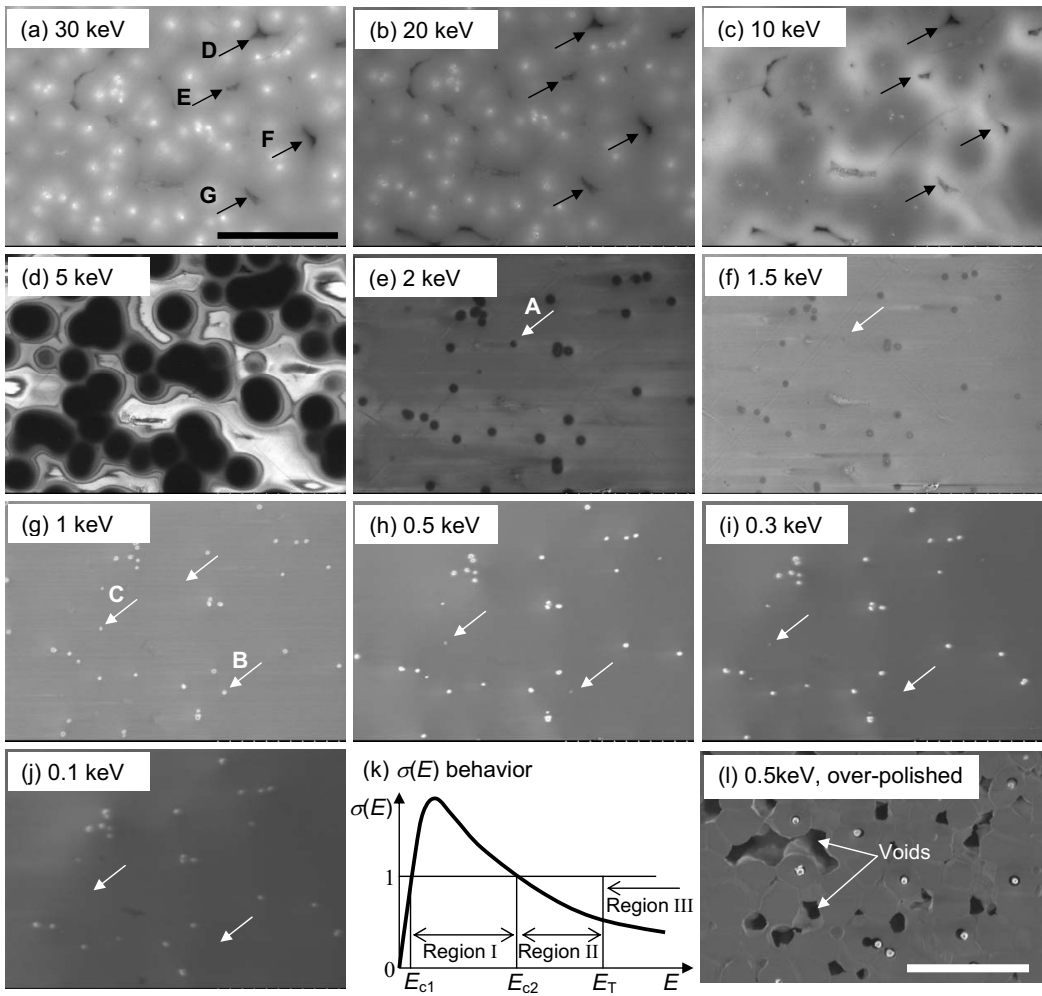

FIG. 2. Secondary electron images of $\mathrm{SiO}_{2}$ top surface with exposed CNF tips with various electron beam energies (E) of (a) $30 \mathrm{keV}$, (b) $20 \mathrm{keV}$, (c) $10 \mathrm{keV}$, (d) 5 $\mathrm{keV}$, (e) $2 \mathrm{keV}$, (f) $1.5 \mathrm{keV}$, (g) $1.0 \mathrm{keV}$, (h) $0.5 \mathrm{keV}$, (i) $0.3 \mathrm{keV}$, and (j) $0.1 \mathrm{keV}$. The imaged areas of these micrographs are the same. Scale bar is $3 \mu \mathrm{m}$. Arrows (A) - (C) shows the unexposed CNFs and arrows (D) (G) indicate the void in $\mathrm{SiO}_{2}$. (k) Schematics of the total electron emission yield $\sigma(E)$ of $\mathrm{SiO}_{2} . E_{\mathrm{c} 1}$ and $E_{\mathrm{c} 2}$ are the cross-over energies where $\sigma(E)$ becomes unity. $E_{T}$ is the energy above which the beam penetrates the $\mathrm{SiO}_{2}$ filling. (1) $\mathrm{SEM}$ image of overpolished $\mathrm{SiO}_{2}$ top surface. Scale bar is $3 \mu \mathrm{m}$.
$<E<5 \mathrm{keV}$ ), where the CNFs and the surrounding part of $\mathrm{SiO}_{2}$ become dark, and region III $(E>10 \mathrm{keV})$, where the CNFs again become bright compared with $\mathrm{SiO}_{2}$. The overall trend of these image contrasts is explained as follows based on voltage contrast mechanisms due to beam-induced charging of $\mathrm{SiO}_{2}$. Development of the charging is described by the total electron emission yield ${ }^{7}$ of $\mathrm{SiO}_{2}, \sigma(E)$, which is defined as the ratio of the number of the emitted electrons to that of incident electrons and shown schematically in Fig. 2(k). The $\sigma(E)$ curve shows a peak at several hundred electron volts, and then gradually decreases with $E$. This peak occurs between two cross-over energies, $E_{\mathrm{c} 1}$ and $E_{\mathrm{c} 2}$, where $\sigma(E)$ becomes unity. When $\sigma(E)>1\left(E_{\mathrm{c} 1}<E<E_{\mathrm{c} 2}\right)$, the $\mathrm{SiO}_{2}$ part is positively charged, forming the potential barrier to reduce the number of emitted SEs. ${ }^{8}$ This leads to a weakened signal emission from $\mathrm{SiO}_{2}$, and in turn, relatively bright signal from the CNF tips, corresponding to region I. Above $E_{\mathrm{c} 2}$, negative charge is developed in $\mathrm{SiO}_{2}$, leading to a relatively dark signal from the CNFs. The negative surface potential can increase $^{8}$ up to the voltage difference between $E$ and $E_{\mathrm{c} 2}$, so that the size of the dark region increases with the increasing beam energy $E_{\mathrm{c} 2}$, as shown in Figs. 2(d)-2(f) in region II. The negative potential buildup then diminishes above the beam energy $E_{T}$ (or region III), which is defined as the energy where the incident electrons start to reach the Ti layer where charge neutralization occurs.

We proceed to discuss the image formation mechanism of the unexposed CNFs. As can be seen in Fig. 2, the number of CNF spots decreases with decreasing beam energy. For example, spot (A) disappears below $1.5 \mathrm{keV}$ and spots (B) and $(\mathrm{C})$ disappear as well below 0.3 and $0.1 \mathrm{keV}$, respectively. This is because higher-energy incident electron has longer penetration depth, and the electrons reaching the embedded CNFs relax the charging of $\mathrm{SiO}_{2}$ residue above the CNF due to the electron beam-induced conductivity, ${ }^{9}$ as illustrated in Fig. 3(a). This means that very low energy beam is required to detect unexposed CNFs so that the beam penetration depth becomes shorter than the thickness of the $\mathrm{SiO}_{2}$ residue, as shown in Fig. 3(b). Based on the beam penetration depth calculation, ${ }^{10}$ the unexposed CNFs (A), (B), and (C) are expected to correspond to $\mathrm{SiO}_{2}$ residual layers of 65 , 4 , and $1 \mathrm{~nm}$ in thickness, respectively, on their tips. Thus one can estimate the residue thickness on unexposed CNFs by changing the beam energy.

Meanwhile, high-energy images in region III [Figs. 2(a)-2(c)] exhibit the dark areas, indicated by arrows (D) (G). Since these dark areas are not visible in regions I and II, these are likely embedded voids. Actually the overpolished $\mathrm{SiO}_{2}$ surface in Fig. 2(1) shows many voids between CNFs, confirming the void detection in Figs. 2(a)-2(c). So far, backscattered electrons (BSEs) have been frequently used for imaging the embedded heavy materials such as copper. ${ }^{11}$ The SE signal, which is used in the present study, however, has been mostly used for surface-sensitive imaging. This is because the captured SEs are mainly produced within the thin surface layer of several nanometers in thickness due to their short mean free path. ${ }^{7}$ One possible technique for subsurface imaging with SE signal is the BSE-induced SEs, ${ }^{7}$ or commonly called as $\mathrm{SE}(2)$, in contrast to $\mathrm{SE}(1)$, which is generated at the point of beam impact. $\mathrm{SE}(2)$ is generated when the BSEs pass through the surface, which are scattered by the deeper atoms, thus the number of $\operatorname{SEs}(2)$ is proportional to the number of BSEs, which can be affected by the volume of the $\mathrm{SiO}_{2}$ layer [Fig. 3(c)]. While this mechanism works well for an embedded material of high atomic number, it exhibits no observable contrast when the BSE coefficients of the embedded structure and its surroundings are similar. ${ }^{7}$ In fact, our previous SEM study ${ }^{12}$ failed to detect the internal nanostructure in horizontal CNFs using the SE signal from the CNF. Thus the SE(2) technique practically does not work for void imaging of light embedded materials as in the present case. 
(a) Low-energy beam

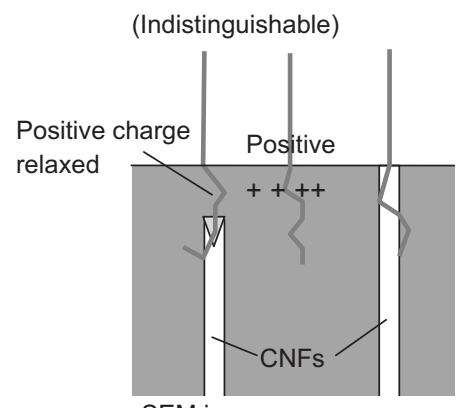

SEM image

(

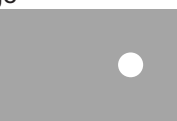

(c) BSE-induced SE model

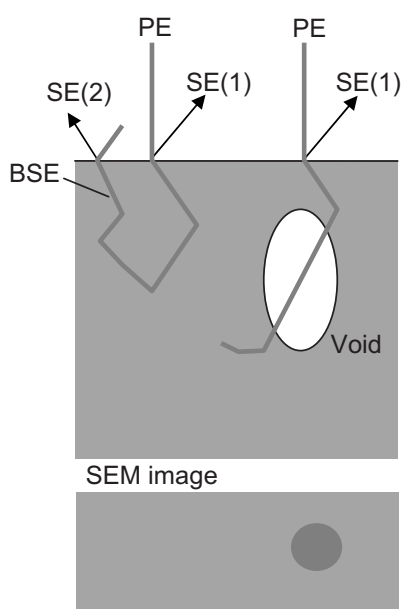

(b) Very-low-energy beam



SEM image

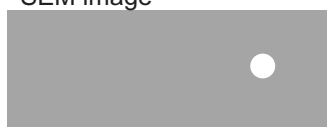

(d) SE-suppression model

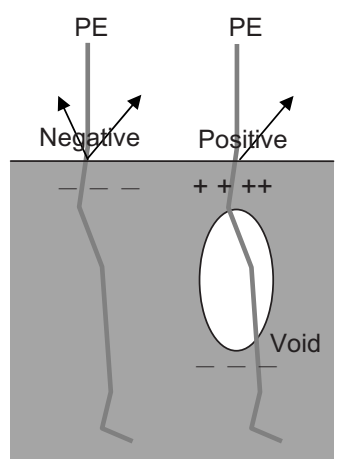

SEM image

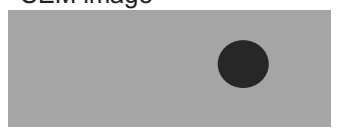

FIG. 3. [(a) and (b)] Schematics of the beam penetration in the thin $\mathrm{SiO}_{2}$ residue on unexposed CNFs (top) and the corresponding SEM images expected (bottom). (c) Backscattered-electron-induced secondary electron [or SE(2)] model of the void detection (top) and the corresponding SEM images (bottom). (d) SE-suppression model of the void detection (top) and the corresponding SEM images (bottom).

We propose an alternative mechanism, which can explain the experimental results based on the recently reported SE suppression phenomena under high-energy electron beam bombardment of a freely supported insulator. ${ }^{13}$ While the electron emission yield of the bulk insulator at $E>E_{\mathrm{c} 2}$ becomes lower than unity [Fig. 2(k)] and the insulator is negatively charged, the freely supported insulating film does not accumulate electrons because most of the incident electrons escape from the backside of the film as transmitted electrons. In this case, additional emission of BSEs and SEs pushes the total emission yield over unity, making the film positively charged. ${ }^{13}$ If we apply this proposed mechanism to the $\mathrm{SiO}_{2}$

layer above the void, the observed dark spots (D)-(G) in Figs. 2(a)-2(c) can be explained as follows. The normal $\mathrm{SiO}_{2}$ regions without voids are negatively charged at $E>E_{\mathrm{c} 2}$ because of $\sigma(E)<1$. The thin $\mathrm{SiO}_{2}$ layer above the void, however, loses electrons due to large electron transmission probability. This leads to positive-charge developed on the surface. As a result, the $\mathrm{SiO}_{2}$ layer above the void shows dark signal intensity compared to the rest of $\mathrm{SiO}_{2}$ as shown in Fig. 3(d), explaining the experimentally observed void images. Since this proposed mechanism is only applied to insulators, it is also consistent with the result in Ref. 12, where the internal structure in CNF was not detected by capturing SE signal from the metallic CNF.

In summary, SEM imaging of defect structures in vertical CNF via interconnects has been presented. Low-energy imaging is shown to be essential to differentiate the unexposed CNF plugs, which can lead to electrical failure. Embedded voids formed in $\mathrm{SiO}_{2}$ layer can be observed using high-energy beam with secondary electron detection. A mechanism of void detection has been proposed based on the recently reported SE suppression phenomena due to positive charging. The image formation analyses presented here are useful for defect inspection of nanoelectronic devices using SEM.

The authors are grateful to Quoc Ngo for sample preparations and valuable discussions and to Bill Roth and Mark Betts of Hitachi High-Technologies, America for technical support in SEM experiments.

${ }^{1}$ A. V. Vairagar, S. G. Mhaisalkar, M. A. Meyer, E. Zschech, A. Krishnamoorthy, K. N. Tu, and A. M. Gusak, Appl. Phys. Lett. 87, 081909 (2005).

${ }^{2}$ W. Steinhögl, G. Schindler, G. Steinlesberger, and M. Engelhardt, Phys. Rev. B 66, 075414 (2002).

${ }^{3}$ M. Nihei, A. Kawabata, D. Kondo, M. Horibe, S. Sato, and Y. Awano, Jpn. J. Appl. Phys., Part 1 44, 1626 (2005).

${ }^{4}$ J. Li, Q. Ye, A. Cassell, H. T. Ng, R. Stevens, J. Han, and M. Meyyappan, Appl. Phys. Lett. 82, 2491 (2003).

${ }^{5}$ Y. Ominami, M. Suzuki, K. Asakura, and C. Y. Yang, Nanotechnology 19, 405302 (2008).

${ }^{6}$ Q. Ngo, T. Yamada, M. Suzuki, Y. Ominami, A. M. Cassell, J. Li, M. Meyyappan, and C. Y. Yang, IEEE Trans. Nanotechnol. 6, 688 (2007).

${ }^{7}$ H. Seiler, J. Appl. Phys. 54, R1 (1983).

${ }^{8}$ X. Meyza, D. Goeuriot, C. Guerret-Piécourt, D. Tréheux, and H.-J. Fitting, J. Appl. Phys. 94, 5384 (2003).

${ }^{9}$ Y. Homma, S. Suzuki, Y. Kobayashi, M. Nagase, and D. Takagi, Appl. Phys. Lett. 84, 1750 (2004).

${ }^{10}$ K. Kanaya and S. Okayama, J. Phys. D 5, 43 (1972).

${ }^{11}$ L. M. Gignac, M. Kawasaki, S. H. Boettcher, and O. C. Wells, J. Appl. Phys. 97, 114506 (2005).

${ }^{12}$ M. Suzuki, Q. Ngo, H. Kitsuki, K. Gleason, Y. Ominami, C. Y. Yang, T. Yamada, A. M. Cassell, and J. Li, J. Vac. Sci. Technol. B 25, 1615 (2007).

${ }^{13}$ M. Suzuki, K. Kumagai, T. Sekiguchi, A. M. Cassell, T. Saito, and C. Y. Yang, J. Appl. Phys. 104, 114306 (2008). 